\title{
Epidemiologic trends in lung cancer over two decades in Northern Greece: an analysis of bronchoscopic data
}

\author{
T. Kontakiotis 1 , N. Manolakoglou2, F. Zoglopitis', D. lakovidis', \\ L. Sacas ${ }^{3}$, A. Papagiannis ${ }^{1}$, A. Mandrali4, D. Papakosta1, \\ P. Argyropoulou2, D. Bouros 5
}

ABSTRACT: Epidemiologic trends in lung cancer over two decades in Northern Greece: an analysis of bronchoscopic data. T. Kontakiotis, N. Manolakoglou, F. Zoglopitis, D. Iakovidis, L. Sacas, A. Papagiannis, A. Mandrali, D. Papakosta, P. Argyropoulou, D. Bouros.

Background and Aim. The relative frequency of histological subtypes of lung cancer in Europe has changed dramatically during the $2^{\text {th }}$ century. The aim of this study was to explore the changing epidemiology of lung cancer in Northern Greece over the last two decades.

Methods. From the extensive database of the Bronchoscopy Unit of the G. Papanicolaou General Hospital, Thessaloniki, Greece, we identified all patients with a histologic and/or cytologic report positive for lung cancer over two consecutive decades.

Results. Between 1/1/1986 and 31/12/2005 we identified 9981 patients with specimens positive for lung cancer. A significant increase in mean patient age was observed during the second decade $(64.8 \pm 9.4$ vs. $62.1 \pm 8.9$, $p=0.001)$. Men developed lung cancer ten times more often than women. The predominant histological type was squamous cell cancer in males $(4203$ cases, $45.7 \%)$ and adenocarcinoma (418 cases, $52.6 \%$ ) in females. The number of lung cancer cases was significantly higher during the second decade compared to the first decade ( 5766 cases $[57.8 \%$ ] vs. 4215 cases [ $42.2 \%$ ], respectively, $p<0.001$ ). There was a significant decrease in the percentage of squamous cell carcinoma in males in the second decade (2317 cases [44.1\%] vs. 1886 cases $[48.0 \%], p<0.001)$, and an increase in adenocarcinoma (1021 cases [19.4\%] vs. $609[11.6 \%$ ],$p<0.001)$. In females, the relative incidence of adenocarcinoma was decreased and that of squamous cell carcinoma was increased, but not significantly. There was no obvious change in the incidence of small cell lung cancer. Neoplastic lesions were most often located in the upper lobes.

Conclusion. The number of lung cancer cases has increased in the last decade. Squamous lung cancer appears to be decreasing in men and increasing in women. Adenocarcinoma appears to be increasing in men and decreasing in women. There appears to be no change in small cell lung cancer. During the second decade there has been a significant decrease in the male: female ratio.

Monaldi Arch Chest Dis 2009; 71: 4, 147-152.

Keywords: Lung cancer, Epidemiology, Bronchoscopy, Adenocarcinoma, Squamous cell carcinoma, Small cell carcinoma.

1 Bronchoscopy Unit, Aristotle University of Thessaloniki,

2 Respiratory Failure Unit, Aristotle University of Thessaloniki,

3 Histopathology Department, G. Papanicolaou General Hospital, Exochi, Thessaloniki

4 Cytology, G. Papanicolaou Geneneral Hospital, Exochi, Thessaloniki,

5 Department of Pneumonology, Medical School Democritus University of Thrace and University Hospital of Alexandroupolis, Alexandroupolis, Greece.

Correspondence: Prof. Theodore Kontakiotis, Dept. of Pneumonology, Medical School, Aristotle University of Thessaloniki, Thessaloniki,Greece,GR-68100; e-mail:kontak@auth.gr

\section{Introduction}

By the end of the $20^{\text {th }}$ century lung cancer had become one of the leading causes of preventable death globally [1]. Its prevalence had increased approximately 10 -fold compared to the previous century, and it continues to be the second most common type of cancer and the leading cause of cancer death in the United States [2]. Its incidence and mortality has also dramatically increased in most European countries, so it is considered as one of the major epidemics of the current century $[3,4]$. Some population based studies show that this epidemic which peaked in the early 1980 s began to decline by the end of the century [5], particularly for squamous cell carcinoma (SqCLC) in males $[6,7]$; however, incidence rates for other types such as adenocarcinoma (ADLC) in males [7, 8] and all types of lung cancer in females continue to rise [6-8]. Evaluation of recent patterns of smoking prevalence in the United States of America indicates that for the next 10 to 15 years lung cancer rates will decrease, but will then level off starting approximately in 2030 [4].

Central airways carcinoma can easily be located and diagnosed after a single inspection with the fiberoptic bronchoscope. It has been reported that forceps biopsy during bronchoscopy provides a 
definite diagnosis in 71 to 93 per cent of cases $[9,10]$ without any serious complication. Unfortunately only a small part of the lungs can be inspected by FOB, and the diagnostic yield of bronchoscopy for peripheral lung tumours ranges between 36 and 52 per cent. Recent data from bronchoscopic examination for peripheral lesions under simultaneous fluoroscopic guidance for cytological or histological sampling, revealed an overall diagnostic yield of 93.4\% [11]. Most of the lung tumours referred for surgical resection or other therapy have bronchoscopic evidence of malignancy, and so this procedure is believed to be the most successful method in the diagnosis of lung cancer $[9,10]$.

To our knowledge there has been no epidemiological study of lung cancer incidence, histology and time trends in Greece. In the present study we present some epidemiological trends of lung cancer in Northern Greece comparing two consecutive decades (1986-1995 vs. 1996-2005) on the basis of bronchoscopic findings with regard to pathology, age and sex distribution, and tumour location.

\section{Patients and methods}

Northern Greece (comprising the regions of Macedonia and Thrace) is a geographical area inhabited by an almost uniform population of about 2.5 million (National Statistical Service of Greece, 2001 population census). Most fiberoptic bronchoscopies (FOB) $(90 \%)$ in this area are performed in a dedicated Bronchoscopy Unit in the George Papanicolaou General Hospital, Thessaloniki, which is the main tertiary referral centre for pulmonary diseases. The Unit has a custom made computer database (based on the FileMaker Pro 7.0 software package [FileMaker, Inc., 2003) of all examinations performed since 1985.

Between 1/1/1986 and 12/31/2005 a total of 32,059 FOBs were performed in the Unit $(26,704$ $83.3 \%$ in males). From the database we identified all the cases with evidence of malignancy on histology and/or cytology. For these patients we extracted data on demographics, tumour location, and pathology. The study had the approval of hospital ethical committee. The results were stratified according to age, tumour location and histology. For analysis purposes we divided the patients into six age groups (AG): AG-I included patients <39 years old, AG-II 40 to 49, AG-III 50 to 59, AG-IV 60 to $69, A G-V 70$ to 79 years and AG-VI $\geq 80$ years old. On the basis of tumour location (according to endoscopic and x-rays findings) and/or site of specimen collection we classified the patients into 10 groups:, 1. Trachea (TR), 2. Right Main Bronchus (RMB), 3. Right Upper Lobe (RUL), 4. Bronchus Intermedius (INT), 5. Right Middle Lobe (RML), 6. Right Lower Lobe (RLL), 7. Left Main Bronchus (LMB), 8. Left Upper Lobe (superior division) (LUL), 9. Lingula (inferior division) (LING) and 10. Left Lower Lobe (LLL).

On the basis of histology the patients were classified according to the 1982 WHO histological classification for lung cancer [12]: 1. Squamous Cell
Cancer (SqCLC), 2. Adenocarcinoma (ADLC), 3. Small Cell Lung Cancer (SCLC), and 4. Other pathology (OTHER). Under 'OTHER' we included cases with large cell carcinoma, mixed-type lung cancer, cases with necrotic malignant tissue and cases without clearly defined histology. Some rare types of malignant, mixed or metastatic tumours were included in the OTHER subgroup but benign tumours were excluded from analysis.

Cases with cancers metastatic to the lungs and those with Papanicolaou Class III cytology were not included in the analysis. In patients with PAP III cytology results the bronchoscopy was repeated. If clinical and bronchoscopic data were negative, they were followed for up to two years. Otherwise they were referred to the thoracic surgery department for thoracoscopy or open lung biopsy. Whenever a discordance was detected between histological and cytological typing (14 out of 9981 cases), the histological result was accepted.

\section{Statistical analysis}

All results were expressed as mean \pm standard deviation (SD). Statistical analyses were performed using the Statistical Program for Social Sciences (SPSS 15.0, SPSS, Inc., Chicago, Illinois, 2006). Group differences were tested for significance using the chi-square test. A $p$ value of $<0.05$ was considered statistically significant. The unpaired $t$-test was used for parametric data, with the Bonferroni adjustment for an alpha level of 0.05 for multiple tests.

\section{Results}

Among a total of 32,059 bronchoscopies, 9981 $(31.1 \%)$ were positive for lung cancer and were included in our analysis. The mean age of all patients was $63.6 \pm 9.3$ years. There were 9187 males (92.0\%, mean age 63.8土9.1 years) and 794 females $(8.0 \%$, mean age, $61.5 \pm 10.8$ years). The male to female ratio was $11.6: 1(p<0.0011)$. In the first decade there were 3928 males $(93.2 \%)$ and 287 females $(6.8 \%)$ (male: female ratio $=13.7 / 1)$, and in the second decade 5259 males $(91.2 \%)$ and 507 females $(8.8 \%$ ) (male: female ratio $=10.4$ ). The decrease in the male-to-female ratio observed during the second decade was statistically significant $(p<0.001)($ table 1$)$.

\section{Age trend analysis}

The age of lung cancer patients ranged from 23 to 96 years. A significant increase in mean age was observed for both sexes in the second decade $(65.05 \pm 9.2$ vs. $62.35 \pm 8.8, p<0.001$ in males and $62.5 \pm 11.4$ vs.59.6 $\pm 9, p<0.001$ in females), and this concerned all histological subtypes (table 2 ). In the first decade we observed a progressive rise in relative lung cancer frequency with advancing age [from $1.6 \%$ of the cases in AG-I ( $<39$ years old) to $47.0 \%$ in AG-IV (60-69 years old) in male as well as in female patients [from $2.4 \%$ in AG-I to $39.0 \%$ in AG-IV $(p<0.001)$, and a decreased frequency 
Table 1. - Distribution of fiberoptic bronchoscopies and lung cancer cases by gender in the two decades

\begin{tabular}{lccc}
\hline $\mathbf{N}$ of FOBs & Male (\%) & Female (\%) & $\boldsymbol{p}$ \\
\hline $1^{\text {st decade }}$ & $12,588(85.5)$ & $2,138(14.5)$ & 0.001 \\
$2^{\text {nd decade }}$ & $14,116(81.4)$ & $3,217(18.6)$ & \\
Total & $26,704(83.3)$ & $5,355(16.7)$ & \\
\hline $\mathbf{N}$ of Cancers & $(\%$ of FOBS $)$ & $(\%$ of FOBS) & 0.001 \\
\hline $1^{\text {st decade }}$ & $3,928(31.2)$ & $287(13.4 \%)$ & \\
$2^{\text {nd decade }}$ & $5,259(37.3)$ & $507(15.8)$ & $794(14.8)$ \\
Total & $9,187(34.4)$ & & \\
\hline
\end{tabular}

FOBs $=$ Fiberoptic bronchoscopies

for both sexes in Age Groups V and VI (figure 1). In the second decade the relative frequency of lung cancer in males increased from $0.7 \%$ in AG-I to $39.4 \%$ in AG-IV and in females from $4.3 \%$ in AGI to $35.7 \%$ in AG-IV. Comparing the two decades we found a significant decrease in lung cancer incidence in the second decade in males compared to the first decade from $1.6 \%$ to $0.7 \%(p<0.001)$ in AG-I, from $24.7 \%$ to $18.4 \%(p<0.001)$ in AG-III, and from $47.0 \%$ to $39.4 \%$ in AG-IV. On the other hand, a significant increase was observed in AG-V and AG-VI (from $19.1 \%$ to $32.8 \%$ and from $1.3 \%$ to $2.7 \%$ ( $p<0.001$, in both instances). In females a decrease was observed during the second decade in AG-III (from $30.7 \%$ to $20.3 \%, p=0.001$ ) and an increase in $\mathrm{AG}-\mathrm{V}$ from $15.0 \%$ to $28.2 \%(p<0.001$ (figure 1).

\section{Trends in histology}

SqCLC was the dominant histological type in males $(45.7 \%$ of all patients, $p<0.001)$, whereas ADLC was the leading type in females (52.6\%, $p<0.001)$. A significant decrease was observed in SqCLC (from $48.0 \%$ to $44.1 \%, p<0.001$ ) with a concurrent increase of ADLC during the second decade in males (from $15.5 \%$ to $19.4 \%, p<0.001$ ). The differences observed in females were not significant, even though there was an increasing trend in SqCLC (from $11.6 \%$ to $18.7 \%$ ) and a decreasing trend in ADLC (from $56.1 \%$ to $50.7 \%$ ). The inci- dence of SCLC and other lung cancer types remained unchanged (table 2). A detailed distribution of histological types according to patient age and sex is shown in table 3 .

\section{Trends in tumour location}

The right upper lobe (RUL) was the most common site of lung cancer in both sexes, and in both decades. The only significant changes between the decades a decrease in lung cancer in males in the $\mathrm{RMB}$ (from $5.0 \%$ to $3.4 \% p<0.01$ ) and in the $\mathrm{LMB}$ (from $6.2 \%$ to $4.8 \%, p=0.003$ ).

\section{Discussion}

Most FOBs in Northern Greece (>90\%) are performed in a dedicated Bronchoscopy Unit in the George Papanicolaou General Hospital, Thessaloniki, which is the main tertiary referral centre for pulmonary diseases. During the second decade bronchoscopy units were developed in some private hospitals, and these accounted for up to $10 \%$ of the cases. As we had no access to their results, we excluded them from our analysis.

In the present epidemiological study comparing two consecutive decades (1986-1995 vs. 19962005), we found that over the last decade the incidence of lung cancer in Northern Greece has bronchoscopically increased in both genders. In terms of histology, squamous cell lung cancer was the

Table 2. - Distribution of histological types of lung cancer by gender in the two decades

\begin{tabular}{|c|c|c|c|c|c|c|c|c|}
\hline & 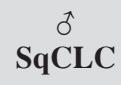 & $\stackrel{\stackrel{9}{+}}{\text { SqCLC }}$ & $\begin{array}{c}\hat{\sigma} \\
\text { ADLC }\end{array}$ & $\begin{array}{c}\stackrel{+}{+} \\
\text { ADLC }\end{array}$ & $\begin{array}{c}\hat{\sigma} \\
\text { SCLC }\end{array}$ & $\stackrel{\stackrel{9}{+}}{\text { SCLC }}$ & $\begin{array}{c}\hat{0} \\
\text { OTHER }\end{array}$ & $\begin{array}{c}\stackrel{q}{q} \\
\text { OTHER }\end{array}$ \\
\hline $1^{\text {st }}$ decade $\mathrm{n}=$ & 1886 & 43 & 609 & 161 & 904 & 35 & 529 & 48 \\
\hline$\%$ & $48,0 \%$ & $15,0 \%$ & $15,5 \%$ & $56,1 \%$ & $23,0 \%$ & $12,2 \%$ & $13,5 \%$ & $16,7 \%$ \\
\hline $2^{\text {nd }}$ decade $n=$ & 2317 & 95 & 1021 & 257 & 1168 & 65 & 753 & 90 \\
\hline$\%$ & $44,1 \%$ & $18,7 \%$ & $19,4 \%$ & $50,7 \%$ & $22,2 \%$ & $12,8 \%$ & $14,3 \%$ & $17,8 \%$ \\
\hline$x^{2}=$ & 14.023 & 1.548 & 23.29 & 1.938 & 0.788 & 0.021 & 1.286 & 0.073 \\
\hline $\mathrm{p}=$ & $<0.001$ & 0.213 & $<0.001$ & 0.164 & 0.375 & 0.886 & 0.257 & 0.788 \\
\hline
\end{tabular}

$\mathrm{SqCLC}=$ squamous cell lung cancer, $\mathrm{ADLC}=$ adenocarcinoma, $\mathrm{SCLC}=$ small cell lung cancer . 


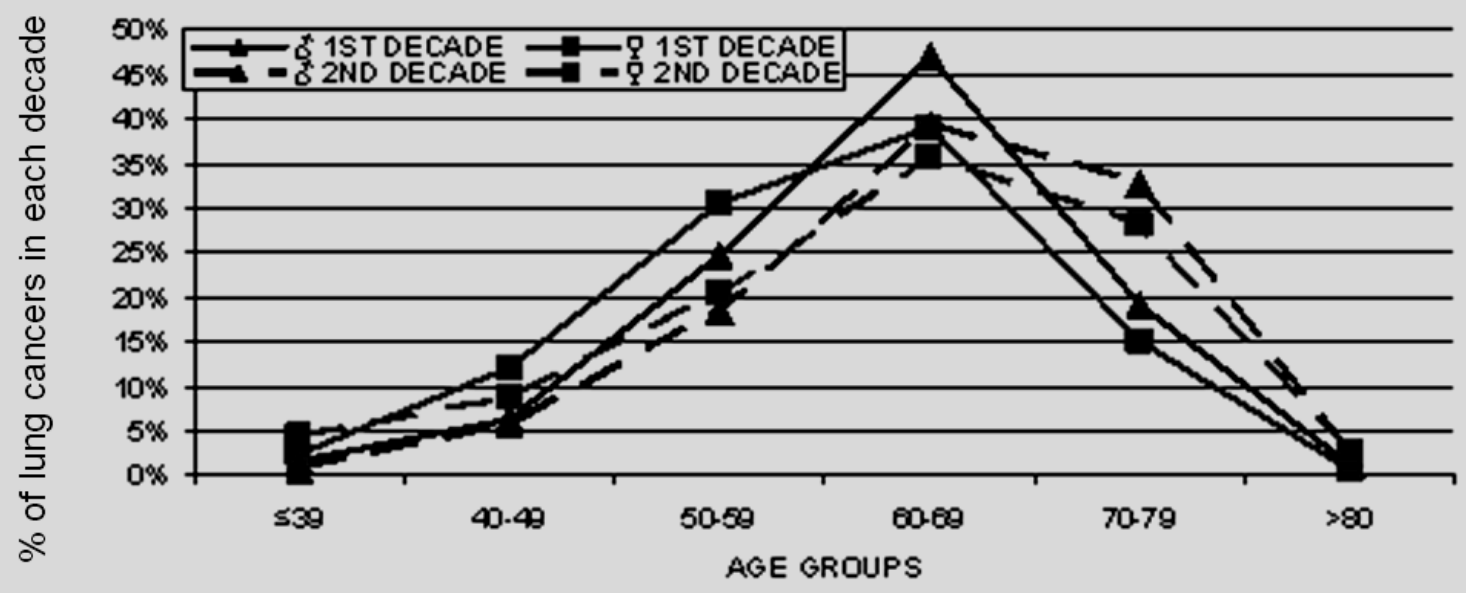

Fig. 1. - Age group analysis of lung cancer patients of both sexes during first and second decade.

predominant cell type in males and adenocarcinoma in females. During the study period, males developed lung tumours ten times more often than females. However, the male to female ratio decreased in the second decade. In terms of tumour location the upper lobes were more frequently involved. The mean age at patient presentation was higher in the second decade.

This was a retrospective epidemiological study of lung cancer in an almost uniform population of approximately 2.5 million, which included 9,981 cancer cases [9,187 male and 794 female] over a 20-year period, representing approximately $90 \%$ of all lung cancer cases in Northern Greece. We speculate that a $10 \%$ of lung cancers are diagnosed elsewhere mainly in other small bronchoscopy centres in the private medicine. We estimated the average annual incidence of lung cancer diagnosed bronchoscopically in this area to be about $0.02 \%$. The male: female ratio was 11.6 (13.6 in the first decade and 10.4 in the second decade). Lung cancer incidence in other countries ranges between $0.012 \%$ (India) and $0.08 \%$ (the Czech Republic [3] for men and between $0.004 \%$ (India) and $0.04 \%$ (Canada and the USA) for women. According to our data lung cancer incidence was about $0.0045 \%$ in females during the first decade and $0.022 \%$ during the second decade. Even though the number of lung cancer cases was doubled in females during the second decade (from 287 to 507 cases), the incidence of lung cancer in women in Northern Greece is still very low. Recent data shows a similarly large preponderance of males in countries neighbouring with Northern Greece such as Macedonia (male: female ratio $=7: 1$ ) [13] and Turkey (M: F = 9.4:1) [14]. We can only speculate as to whether these similarities are due to analogous smoking habits in the two genders or are related to other factors. In unpublished data from our bronchoscopic department $68 \%$ of the males bronchoscoped were smokers (20-60 pack-years) with a stabilized time trend, whereas only $10 \%$ of females are smokers (10-30 pack-years) with an increasing trend especially in younger subjects.
Our study shows that over the last decade there has been a significant increase in lung cancer incidence both in males and in females compared to the previous decade. Recently, lung cancer incidence in males was found to have decreased since the 1980s in northern European countries, although in other European countries it had been increasing until the 1990s [3]. Among females the incidence of lung cancer had not peaked in the 1990s either in Europe or in the USA [2]. In contrast, a decline in lung cancer incidence in males has been observed in the USA since the 1990s [7].

Our study was conducted in Northern Greece, where environmental factors have not changed over the years and no exposure to radon or uranium has been detected (there are no nuclear facilities in Northern Greece, and practically all buildings are well aerated). The predominant histological types were SqCLC in males $(45.7 \%)$ and ADLC in females (52.6\%). In male patients, the proportion of ADLC increased and that of SqCLC decreased in the second decade. SqCLC has remained constant in males in the USA over the previous decades while the incidence of ADLC has increased. In female patients the rate of ADLC showed minimal decrease during the second decade, whereas that of SqCLC increased, though not significantly. This decrease of $\%$ adenocarcinoma in our study is obviously because of the \% increase of squamous cell carcinoma, because of the changes in female smoking habits. In females in the USA, ADLC has shown minimal increase, but the incidence of SqCLC, and especially of SCLC, has increased significantly [7].

Ikeda et $\mathrm{al}$, in a review of 1151 cases from a University hospital between 1970 and 1989 [15] reported that the proportion of ADLC increased from 26 to $45 \%$ in males and from 45 to $69 \%$ in females. However, some surveys have failed to show this change: a study from the Tumour Registry of New Mexico, USA, showed an increasing proportion of SCLC rather than ADLC among the female population, but a histological diagnosis was only available in half of the cases. A study from Min- 
Table 3. - Distribution of histological types of lung cancer by gender and patient age in the two decades

Distribution of histological types of lung cancer in both sexes according to patient age; comparison of two decades

\begin{tabular}{|c|c|c|c|c|c|c|c|c|}
\hline $\begin{array}{l}\text { Age (AG) } 1 \\
\text { LCS }^{2}\end{array}$ & 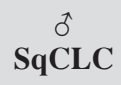 & $\stackrel{\stackrel{9}{7}}{\text { Sq }} \stackrel{\text { CLC }}{ }$ & $\begin{array}{c}\hat{0} \\
\text { ADLC }\end{array}$ & $\begin{array}{c}\stackrel{+}{+} \\
\text { ADLC }\end{array}$ & $\begin{array}{c}\hat{0} \\
\text { SCLC }\end{array}$ & $\begin{array}{c}\stackrel{9}{+} \\
\text { SCLC }\end{array}$ & $\begin{array}{c}\text { ô } \\
\text { OTHER }\end{array}$ & $\stackrel{\stackrel{q}{+}}{\text { OTHER }}$ \\
\hline$\leq 39$ (I) $\quad 1^{\text {st }}$ & $1.1 \%$ & $0.0 \%$ & $2.0 \%$ & $2.5 \%$ & $1.9 \%$ & $0.0 \%$ & $2.5 \%$ & $6.3 \%$ \\
\hline$\leq 39$ (I) $\quad 2^{\text {nd }}$ & $0.4 \%$ & $3.2 \%$ & $0.9 \%$ & $2.7 \%$ & $1.1 \%$ & $0.0 \%$ & $0.9 \%$ & $13.3 \%$ \\
\hline $\mathbf{p}=$ & 0.015 & 0.584 & 0.097 & 0.869 & 0.206 & N/A & 0.052 & 0.324 \\
\hline $40-49$ (II) $\quad 1^{\text {st }}$ & $5.1 \%$ & $16.3 \%$ & $6.9 \%$ & $9.3 \%$ & $7.5 \%$ & $25.7 \%$ & $7.2 \%$ & $8.3 \%$ \\
\hline $40-49$ (II) $\quad 2^{\text {nd }}$ & $4.3 \%$ & $9.5 \%$ & $7.5 \%$ & $9.3 \%$ & $6.6 \%$ & $6.2 \%$ & $7.4 \%$ & $7.8 \%$ \\
\hline $\mathbf{p}=$ & 0.209 & 0.385 & 0.7 & 0.869 & 0.462 & 0.014 & 0.95 & 0.83 \\
\hline $50-59(\mathrm{III}) \quad 1^{\mathrm{st}}$ & $22.8 \%$ & $27.9 \%$ & $25.0 \%$ & $31.1 \%$ & $28.9 \%$ & $28.6 \%$ & $24.4 \%$ & $33.3 \%$ \\
\hline $50-59$ (III) $\quad 2^{\text {nd }}$ & $16.4 \%$ & $18.9 \%$ & $20.6 \%$ & $19.5 \%$ & $21.1 \%$ & $30.8 \%$ & $17.7 \%$ & $16.7 \%$ \\
\hline $\mathbf{p}=$ & 0.001 & 0.338 & 0.045 & 0.01 & 0.001 & 1 & 0.004 & 0.043 \\
\hline $60-69$ (IV) $\quad 1^{\text {st }}$ & $48.7 \%$ & $34.9 \%$ & $45.5 \%$ & $44.1 \%$ & $44.0 \%$ & $28.6 \%$ & $47.4 \%$ & $33.3 \%$ \\
\hline $60-69$ (IV) $\quad 2^{\text {nd }}$ & $39.5 \%$ & $32.6 \%$ & $36.4 \%$ & $35.8 \%$ & $41.5 \%$ & $40.0 \%$ & $39.8 \%$ & $35.6 \%$ \\
\hline $\mathbf{p}=$ & 0.001 & 0.948 & 0.001 & 0.112 & 0.272 & 0.359 & 0.008 & 0.941 \\
\hline $70-79(\mathrm{~V}) \quad 1^{\text {st }}$ & $20.5 \%$ & $20.9 \%$ & $18.9 \%$ & $11.8 \%$ & $17.0 \%$ & $17.1 \%$ & $18.1 \%$ & $18.8 \%$ \\
\hline $70-79(\mathrm{~V}) \quad 2^{\text {nd }}$ & $36.2 \%$ & $30.5 \%$ & $32.1 \%$ & $29.6 \%$ & $27.3 \%$ & $23.1 \%$ & $31.7 \%$ & $25.6 \%$ \\
\hline $\mathbf{p}=$ & 0.001 & 0.336 & 0.001 & 0.001 & 0.001 & 0.662 & 0.001 & 0.49 \\
\hline$\geq 80(\mathrm{VI}) \quad 1^{\mathrm{st}}$ & $1.7 \%$ & $0.0 \%$ & $1.8 \%$ & $1.2 \%$ & $0.7 \%$ & $0.0 \%$ & $0.4 \%$ & $0.0 \%$ \\
\hline$\geq 80(\mathrm{VI}) \quad 2^{\text {nd }}$ & $3.2 \%$ & $5.3 \%$ & $2.4 \%$ & $3.1 \%$ & $2.3 \%$ & $0.0 \%$ & $2.4 \%$ & $1.1 \%$ \\
\hline $\mathbf{p}=$ & 0.004 & 0.298 & 0.497 & 0.394 & 0.005 & N/A & 0.008 & 0.748 \\
\hline
\end{tabular}

SqCLC $=$ squamous cell lung cancer, $\mathrm{ADLC}=$ adenocarcinoma, $\mathrm{SCLC}=$ small cell lung cancer. ${ }^{1}=$ Age Group, ${ }^{2}=$ Lung Cancer Subtypes, $1^{\text {st }}=1^{\text {st }}$ decade $(1986-1995), 2^{\text {nd }}=2^{\text {nd }}$ decade $(1996-2005)$ in males or females in each decade. $N / A=$ not applicable.

nesota, USA, showed an equal increase in all histological cell types of bronchogenic carcinoma [17].

It is of interest that the relative frequency of SqCLC continued to increase steadily in males with increasing age, from $28 \%$ in patients $<39$ years to $53 \%$ in patients $>80$ years old. In females, the relative frequency of ADLC was about $50 \%$ in the 40-50 AG and remained constant up to the $>80$ AG. Age analysis of lung cancer histological subtypes revealed that patients with ADLC were younger than those with SqCLC in both genders. Similar results have been reported by Choi et al [18] who showed, in their study of 2,229 patients, a predominance of ADLC in the young AG $(<39$ years old).

The mean age of our male patients was higher than that of female patients (64.7 vs $62.4 \mathrm{yrs})$. This is different from other studies $[13,14,16]$ which found that the mean age of women at the time of tumour diagnosis is higher than that of men.
Finally, in this study we recorded the location of the malignancy. The right upper lobe was the most common tumour site, followed by the left upper lobe. These findings are in keeping with the literature $[13,19,20]$, and the only plausible explanation for this predilection is that the upper lobes have been shown to be more affected by tobacco smoke [21].

The higher incidence of lung cancer in patients with connective tissue diseases and the changes in smoking habits should be recognised $[22,23]$. We were unable to collect smoking habits data for 20 years period.

The main limitation of our study was that it only included the bronchoscopic proved lung cancer. The diagnosis of cancer has changed the last decade due to the increased usage of other diagnostic procedures (mediastinoscopy, fine needle aspiration, VATS (video assisted thoracoscopy etc) as well as new methods such as new cancer indexes, PET scan etc). It has therefore been difficult to 
compare these two decades. Conversely every effort has been made from the investigation team to follow up these patients for more than two years and explain the causes of death in most of the investigated patients. According to a recent study of our department [24], over a 30-month period 113 patients with SPN were examined in our department and $11 \%$ of them had positive cytologic and/or pathologic findings of malignancy. We included these patients in our statistics but we excluded patients diagnosed with other methods such as fine needle aspiration or thoracoscopy because we had no data especially for the first decade examined. A statistically significant increase in diagnostic yield was observed when bronchoscopy was performed under fluoroscopy guidance (from $11.5 \%$ in initial bronchoscopy to $62.5 \%, x^{2}=19.65$, $p=0.0001$ ) [23]. Bronchoscopy under fluoroscopy guidance was mainly performed in our department in the second decade, so for statistical purposes data obtained by this method was not included in our analysis.

Our data accords with Reichenberger et al [25]. In this series only $5 \%$ of all bronchoscopies were performed for SPN. The use of TBNA under fluoroscopy guidance increased the diagnostic yield of bronchoscopy from 35 to $51 \%$.

In conclusion, we observed that over the past decade the incidence of bronchoscopically diagnosed lung cancer in Northern Greece has increased in both genders. During the study period, males developed lung tumours ten times more often than females. The neoplastic lesions were most commonly located in the upper lobes. The most frequent cell type was squamous carcinoma in males and adenocarcinoma in females.

Acknowledgements. We would like to thank Miss Elli Lithoxopoulou, health psychologist in our department, for careful revision of the manuscript, and Associate Professor Theodore Konstantinidis for statistical and epidemiological advice.

\section{References}

1. Rosen G. History of Public Health. Science 1959; 23: 129 (3343): 236.

2. Page GP, Green JL, Lackland D. Epidemiology of lung cancer with special reference to genetics, bioassays, women, and developing countries. Semin Respir Crit Care Med 2000; 21: 365-73.

3. Janssen-Heijnen ML, Coebergh JW. The changing epidemiology of lung cancer in Europe. Lung Cancer 2003; 41: 245-58.

4. Alberg AJ, Brock MV, Samet JM. Epidemiology of lung cancer: looking to the future. J Clin Oncol 2005; 23: $3175-85$.

5. Centers for Disease Control and Prevention (CDC). Declines in lung cancer rates - California, 1988-1997. MMWR Morb Mortal Wkly Rep 2000; 49: 1066-9.

6. Cancer Stat Fact Sheets Cancer of the Lung and Bronchus NCI Issues Cancer Trends Progress Report: 2007 Update National Cancer Institute U.S.
7. Devesa SS, Bray F, Vizcaino AP, Parkin DM. International lung cancer trends by histologic type: male: female differences diminishing and adenocarcinoma rates rising. Int J Cancer 2005; 117: 294-9.

8. Bilello KS, Murin S, Matthay RA. Epidemiology, etiology, and prevention of lung cancer. Clin Chest Med 2002; 23:1-25.

9. Prakash UB. Advances in bronchoscopic procedures. Chest 1999; 116: 1403-8.

10. Postmus P. Bronchoscopy for lung cancer. Chest 2005; 128: 16-18.

11. Kawaraya M, Gemba K, Ueoka H, et al. Evaluation of various cytological examinations by bronchoscopy in the diagnosis of peripheral lung cancer. Br J Cancer 2003; 89: 1885-8.

12. Brambilla E, Travis WD, Colby TV, Corrin B, Shimosato Y. The new World Health Organization classification of lung tumours. Eur Respir J 2001; 18: 1059-68.

13. Pavlovska I, Danilovski D, Orovchanec N, Stefanovski $\mathrm{T}$, Taushanova B, et al. An epidemiologic study of some characteristics of lung cancer. Folia Med (Plovdiv) 2004; 46: 23-31.

14. Goksel T, Akkoclu A; Turkish Thoracic Society, Lung and Pleural Malignancies Study Group. Pattern of lung cancer in Turkey, 1994-1998. Respiration 2002; 69: 207-10.

15. Ikeda T, Kurita Y, Inutsuka S, Tanaka K, Shigematu T.The changing pattern of lung cancer by histologic type-a review of 1151 cases from a university hospital in Japan, 1970-1989. Lung Cancer 1991; 7: 157-764.

16. Butler C, Samet JM, Humble CG, Sweeney ES. Histopathology of lung cancer in New Mexico 1970-72 and 1980-81 1987. J Natl Cancer Inst 1987; 78: 85-90.

17. Beard CM, Jedd MB, Woolner LB, Richardson RL, Bergstralh EJ, Melton LJ. Fifty-year trend in incidence rates of bronchogenic carcinoma by cell type in Olmsted County, Minnesota. J Natl Cancer Inst 1988; 80: 1404-7.

18. Choi JH, Chung HC, Yoo NC, et al. Changing trends in histologic types of lung cancer during the last decade (1981-1990) in Korea: a hospital-based study. Lung Cancer 1994; 10: 287-96.

19. Celikoglu SI, Aykan TB, Karayel T, Demirci S, Goksel FM. Frequency of distribution according to histological types of lung cancer in the tracheobronchial tree. Respiration 1986; 49: 152-6.

20. Levi F, Franceschi S, La Vecchia C, Randimbison L, Te VC. Lung carcinoma trends by histologic type in Vaud and Neuchâtel, Switzerland, 1974-1994. Cancer 1997; 79: 906-14.

21. Hoffmann D, Hoffmann I. The changing cigarette, 1950-1995. J Toxicol Environ Health 1997; 50: 307-64.

22. Adzić TN, Pesut DP, Nagorni-Obradović LM, et al. Clinical features of lung cancer in patients with connective tissue diseases: a 10-year hospital based study. Respir Med 2008; 1 02: 620-4.

23. Kubina M, Hédelin G, Charloux A, Purohit A, Pauli G, Quoix E. Do patients with squamous cell carcinoma or adenocarcinoma of the lung have different smoking histories? Rev Mal Respir 1999; 16: 539-49.

24. Zoglopitis F. Peripheral pulmonary shadowing estimation of diagnostic procedures. Doctoral thesis, Aristotle University of Thessaloniki 2006.

25. Reichenberger F, Weber J, Tamm M, et al. The value of transbronchial needle aspiration in the diagnosis of peripheral pulmonary lesions. Chest 1999; 116: 704-8. 